\title{
Optimal Integral Force Feedback and Structured PI Tracking Control: Application for Objective Lens Positioner.
}

\author{
Yik R. Teo ${ }^{\mathrm{a}, *}$, Douglas Russell ${ }^{\mathrm{b}}$, Sumeet S. Aphale ${ }^{\mathrm{b}}$, Andrew J. Fleming ${ }^{\mathrm{a}}$ \\ ${ }^{a}$ Precision Mechatronics Lab, School of Electrical Engineering and Computer Science, The University of Newcastle, Callaghan, NSW 2308, Australia. \\ ${ }^{b}$ Centre for Applied Dynamics Research, School of Engineering, University of Aberdeen, Aberdeen, AB24 3FX, United Kingdom.
}

\begin{abstract}
This paper describes a new vibration damping technique based on Integral Force Feedback (IFF). Classical IFF utilises a force sensor and integral controller to damp the resonance modes of a mechanical system. However, the maximum modal damping depends on the frequency difference between the system's poles and zeros. If the frequency difference is small, the achievable modal damping may be severely limited. The proposed technique allows an arbitrary damping ratio to be achieved by introducing an additional feed-through term to the control system. This results in an extra degree of freedom that allows the position of the zeros to be modified and the maximum modal damping to be increased. The second contribution of this paper is a structured PI tracking controller that is parameterized to cancel the additional pole introduced by integral force feedback. The parameterized controller has only one tuning parameter and does not suffer from reduced phase margin. The proposed techniques are demonstrated on a piezoelectric objective lens positioner. The results show exceptional tracking and damping performance while maintaining insensitivity to changes in resonance frequency. The maximum bandwidth achievable with a commercial PID controller is $26.1 \mathrm{~Hz}$. In contrast, with the proposed damping and tracking controller, the bandwidth is increased to $255 \mathrm{~Hz}$.
\end{abstract}

Keywords: Force Feedback, Damping Control, Tracking Control, Nanopositioning

\section{Introduction}

High-speed precision positioners are widely used in applications such as confocal microscopes [1], scanning probe microscopy [2, 3], nanofabrication [4] and electrical characterization of semiconductors [5]. A typical nanopositioning system is illustrated in Figure 1. One difficulty with nanopositioning systems is the mechanical resonances that arise from the interaction between the platform mass and flexures, mechanical linkages and actuators. As a result, the frequency of the driving signal for instance a triangular reference is commonly limited to $1 \%-10 \%$ of the resonance frequency to avoid excitation of the mechanical resonance. In commercial nanopositioning systems the most common type of control is sensor-based feedback control using proportional integral or integral controllers. The benefits of these controllers include robustness to modelling error, simplicity of implementation and reduced piezoelectric nonlinearity due to a high loop gain at low frequency. However, the bandwidth of an integral tracking controller $C_{t}(s)=K / s$ is limited by the presence of highly resonant modes. In references $[6,7]$ it was shown that the maximum closed-loop bandwidth is $2 \zeta \omega_{n}$, where $\zeta$ is the damping ratio and $\omega_{n}$ is the natural frequency. Since the damping ratio is usually in the order

\footnotetext{
*Principal corresponding author

Email addresses: yik.teo@ newcastle.edu. au (Yik R. Teo), r01dr12@abdn.ac.uk (Douglas Russell), s.aphale@abdn.ac.uk (Sumeet S. Aphale), andrew. fleming@newcastle.edu.au (Andrew J. Fleming)
}

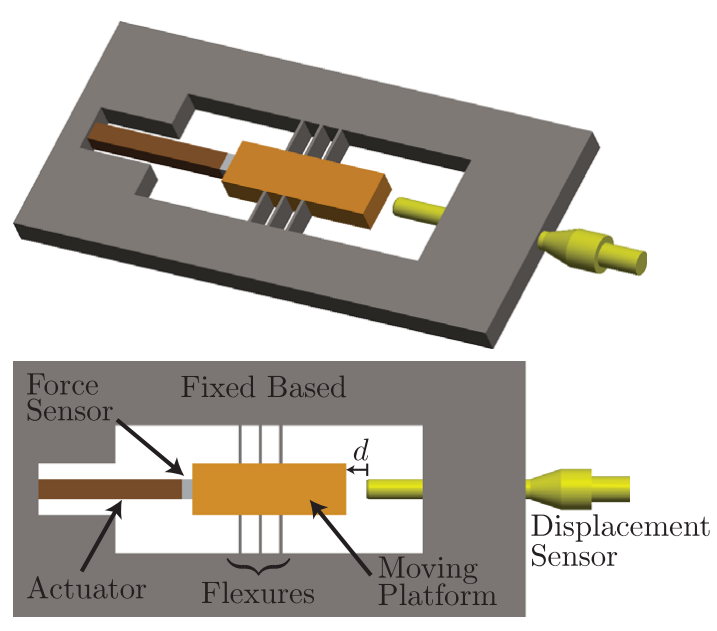

Figure 1: Single-degree-of freedom positioning stage.

of 0.01 , the maximum closed-loop bandwidth is less than $2 \%$ of the resonance frequency.

To improve the closed-loop bandwidth of nanopositioning systems, techniques such as notch filters or plant inversion filters can be implemented [8]. Such techniques can provide significantly improved closed-loop bandwidth provided an accurate model of the system is available. Therefore, notch or plant inversion filters are most practical in systems with stable resonance frequencies or where the feedback controller can be continually recalibrated. On the other hand, model-based control such as robust $\mathscr{H}_{\infty}$ controllers [9] and LMI based controllers 
[10] have also been successfully applied to control such systems.

An alternative method to improve the closed-loop response is damping control. Damping controllers have the advantage of being insensitive to variations in resonance frequency. Furthermore, it has been shown that damping controllers provide better external disturbance rejection than inversion-based systems [11]. A number of techniques for damping control have been successfully demonstrated in the literature. These include Positive Position Feedback (PPF) [12], polynomial based control [13], acceleration feedback [14], shunt control [15, 16], resonant control [17], and Integral Resonance Control (IRC) $[18,19]$. Among these techniques, PPF controllers, velocity feedback controllers, force feedback controllers, and IRC controllers have been shown to guarantee stability when the plant is strictly negative imaginary [20].

Integral force feedback (IFF) a damping control technique described in references [6, 21-25]. The advantages of IFF are the simplicity of the controller, guaranteed stability and excellent performance robustness. Furthermore, IFF can also be implemented using an analog filter. However, one of the limitations of IFF is that the maximum modal damping depends on the frequency difference between the system's poles and zeros. If the frequency difference is small, the achievable modal damping may be severely limited. Furthermore, when the IFF system is enclosed in a tracking loop , the closed-loop performance is limited by an additional pole introduced by the integral force feedback controller.

In this work, we proposed a technique that allows an arbitrary damping ratio to be achieved by introducing an additional feed-through term to the control system. This allows the position of the zeros to be modified, hence, increasing the maximum modal damping. Furthermore, we identified the additional pole that is introduced by the integral force feedback controller and compensate it by parameterising the tracking controller with a zero that cancels the additional pole.

The remainder of the paper is organised as follows. In Section 2, the modelling of a single-degree-of-freedom positioning system is shown. Section 3 compares the proposed damping control technique with classical integral force feedback control. The tracking controller designs are discussed in Section 4. A simulation example is shown Section 5 follow by the experimental result on a commercial objective lens positioner in Section 6.

\section{Modelling a Nanopositioning System}

The single-degree-of-freedom positioner illustrated in Figure 2 can be represented by a second-order mechanical system. The equation of motion for this system is

$$
M_{p} \ddot{d}+c_{f} \dot{d}+\left(K_{a}+k_{f}\right) d=F_{a},
$$

where $M_{p}$ is the mass of the platform and the stiffness and damping coefficient of the flexures are denoted by $k_{f}$ and $c_{f}$ respectively. The force applied by the actuator is $F_{a}$ and the

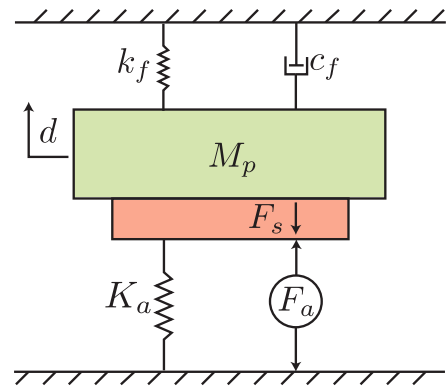

Figure 2: Mechanical diagram of a single-degree-of freedom positioner where $F_{S}$ is the measured force acting between the actuator and the mass of the platform in the vertical direction.

actuator stiffness is $K_{a}$. A force sensor is collocated with the actuator and measures the load force, $F_{s}$.

The configuration of the system is such that the actuator and flexure appear mechanically in parallel, hence, the stiffness coefficients can be grouped together, $k=K_{a}+k_{f}$ which simplifies the equation of motion (1) to

$$
M_{p} \ddot{d}+c_{f} \dot{d}+k=F_{a} .
$$

The transfer function from actuator force, $F_{a}$, to the displacement of the platform, $d$ is

$$
G_{d F_{a}}(s)=\frac{d}{F_{a}}=\frac{1}{M_{p} s^{2}+c_{f} s+k} .
$$

The sensor force, $F_{s}$, can be written as

$$
\begin{aligned}
F_{s} & =F_{a}-d K_{a}, \\
& =F_{a}-K_{a} F_{a} G_{d F_{a}}(s), \\
& =F_{a}\left(1-K_{a} G_{d F_{a}}(s)\right) .
\end{aligned}
$$

The transfer function between the applied force, $F_{a}$, and measured force, $F_{S}$, is found by rearranging (4).

$$
G_{F_{s} F_{a}}(s)=\frac{F_{s}}{F_{a}}=1-K_{a} G_{d F_{a}}(s) .
$$

The force developed by the actuator, $F_{a}$, is

$$
F_{a}=K_{a} \delta
$$

where $\delta$ is the unconstrained piezo expansion.

Substituting (6) into (5), we obtain the transfer function from the unconstrained piezo expansion $\delta$ to the force of the sensor $F_{S}$

$$
G_{F_{s} \delta}=\frac{F_{s}}{\delta}=K_{a} \frac{F_{s}}{F_{a}}=K_{a}\left(1-K_{a} G_{d F_{a}}(s)\right) .
$$

A valid assumption is that the effect of the damping in the flexure, $c_{f}$, is small and thus negligible. The frequency of the openloop poles $\omega_{1}$ and zeros $z_{1}$ of (5) are

$$
\omega_{1}=\sqrt{\frac{k}{M_{p}}}=\sqrt{\frac{K_{a}+k_{f}}{M_{p}}} \quad z_{1} \sqrt{\frac{k_{f}}{M_{p}}} .
$$




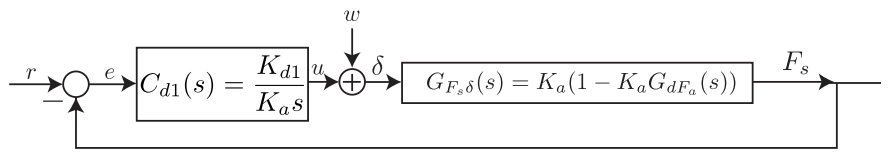

(a) Classical Integral Force Feedback.

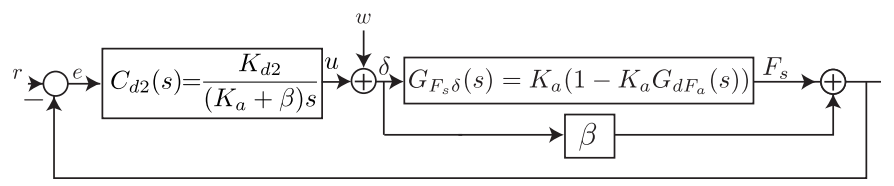

(b) Optimal Integral Force Feedback with new feedthrough term $\beta$.

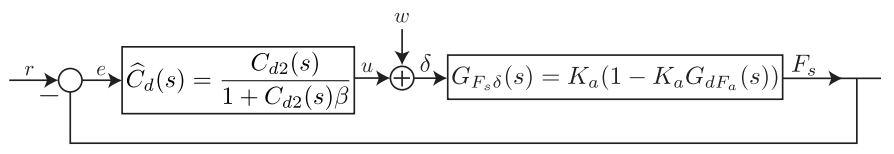

(c) Optimal Integral Force Feedback with new equivalent controller.

Figure 3: Damping Control: Integral Force Feedback Block Diagrams.

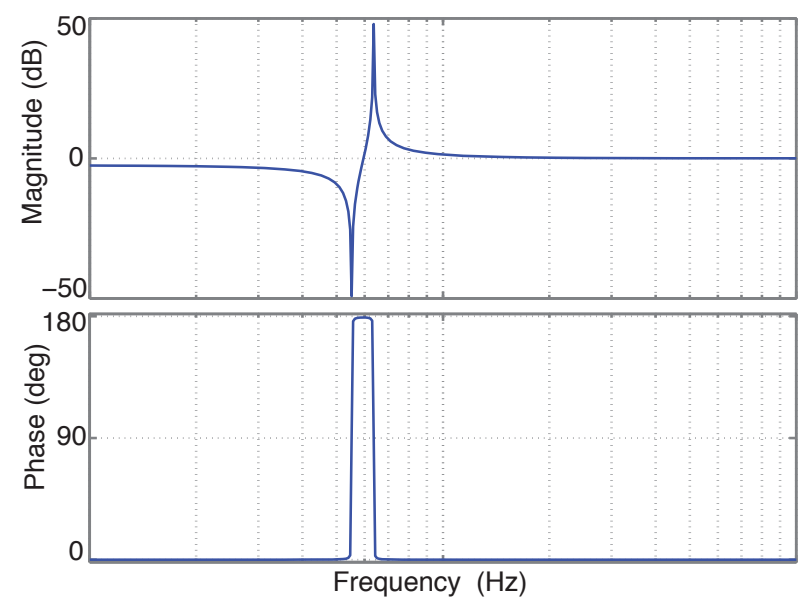

Figure 4: Typical frequency response of $G_{F_{s} F_{a}}(s)$.

\section{Damping Control}

Integral force feedback (IFF) is a popular method for damping control, as described in references [6, 21-25]. This technique utilizes a force sensor and integral controller to directly augment the damping of a mechanical system. The major advantages of IFF is the simplicity of the controller, guaranteed stability, excellent performance robustness, and the ability to damp a large number of resonance modes with a first order controller. Moreover, a piezoelectric force sensor has significantly lower noise density as compared to inductive or resistive strain sensors [26].

\subsection{Classical Integral Force Feedback}

The technique of Classical Integral Force Feedback (CIFF) has been widely applied for augmenting the damping of flexible structures. The feedback law is simple to implement and, under common circumstances, provides excellent damping performance with guaranteed stability.

The open loop transfer function between the unconstrained piezo expansion $\delta$ to the sensor force $F_{s}$ is adapted from Ref.[22]

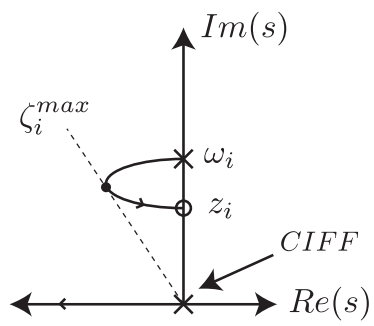

(a) Classical method.

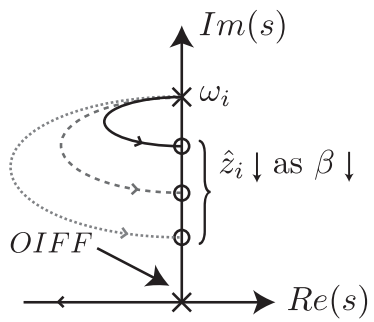

(b) Optimized method.
Figure 5: Damping Control: Typical Root Locus Plots

$$
G_{F_{s} \delta}(s)=\frac{F_{s}}{\delta}=K_{a}\left\{1-\sum_{i=1}^{n} \frac{v_{i}}{1+s^{2} / \omega_{i}^{2}}\right\},
$$

where the sum extends to all the modes, $\omega_{i}$ is the natural frequency of the system and $v_{i}$ is the fraction of modal strain energy for the $i^{t h}$ mode. The corresponding zeros of each mode is given as $z_{i}^{2}=\omega_{i}^{2}\left(1-v_{i}\right)$ [22].

For the positioning application the first resonance mode is of significant interest, this reduce (9) to a second order system (7) . The feedback diagram of an IFF damping controller is shown in Figure 3(a). The frequency response of $G_{F_{s} F_{a}}$ is shown in Figure 4. A key observation of the system $G_{F_{s} F_{a}}$ is that its phase response lies between 0 and $180^{\circ}$. This is a general feature of flexible structures with inputs and outputs proportional to applied and measured forces. A unique property of such systems is that integral control can be directly applied to achieve damping, i.e.

$$
C_{d 1}(s)=\frac{K_{d 1}}{K_{a} s},
$$

where $K_{d 1}$ is the damping control gain. As the integral controller has a constant phase lag of $90^{\circ}$, the loop-gain phase lies between -90 and $90^{\circ}$. That is, the closed-loop system has an infinite gain margin and phase margin of $90^{\circ}$. Simplicity and robustness are two outstanding properties of systems with CIFF.

A solution for the optimal feedback gain has already been derived in [22]. These results can be directly adapted for the system considered in this study. The method makes the valid assumption that system damping coefficients are small and can be neglected. With these assumptions, the maximum modal damping is [22]

$$
\zeta_{i}^{\max }=\frac{\omega_{i}-z_{i}}{2 z_{i}},
$$

and is achieved for

$$
K_{d 1}=\omega_{i} \sqrt{\frac{\omega_{i}}{z_{i}}} .
$$

The root locus plot corresponding to CIFF is shown in Figure 5(a). Note that a key characteristic of this system is that the position of the poles and zeros alternates. The main limitation of the classical method is that the maximum modal damping (11) depends on the distance between the system poles $\omega_{i}$ and modal zeros $z_{i}$. If the distance between the pole and zero 
is small, the maximum modal damping achievable with CIFF is reduced. This means that some systems can be critically damped using CIFF while other systems exhibit insufficient damping.

\subsection{Optimal Integral Force Feedback}

Here, we discuss an extension to the classical technique of Integral Force Feedback that allows an arbitrary damping ratio to be achieved for any system. A new feed-through term $\beta$ is introduced into the system as shown in Figure 3(b). The location of the modal zeros is given as

$$
\widehat{z}_{i}(\beta)=\sqrt{\omega_{i}^{2}\left(1-\frac{K_{a}}{K_{a}+\beta} \frac{K_{a}}{k}\right)} .
$$

This results in an extra degree of freedom that allows the position of the zeros to be modified. As $\beta$ decreases, the zeros of the system will move closer to the real axis, under the condition that $K_{a}\left(v_{i}-1\right)<\beta<0$ is satisfied.The new maximum damping ratio of the system is given as

$$
\widehat{\zeta}_{i}^{\max }=\frac{\omega_{i}-\widehat{z}_{i}(\beta)}{2 \widehat{z}_{i}(\beta)},
$$

The controller is given as

$$
C_{d 2}(s)=\frac{K_{d 2}}{\left(K_{a}+\beta\right) s} .
$$

The corresponding optimal gain is given as

$$
K_{d 2}=\omega_{i} \sqrt{\frac{\omega_{i}}{\widehat{z}_{i}(\beta)}} .
$$

Given a desired damping ratio $\zeta_{d}<1$, the expression for $\beta$ is found by replacing (13) into (14) and rearranging the equation as

$$
\beta=-K_{a}+\frac{K_{a} v_{i}\left(2 \zeta_{d}+1\right)^{2}}{4 \zeta_{d}\left(1+\zeta_{d}\right)} .
$$

where $v_{i}=K_{a} / k$ for the nanopositioning system in Section 2 . The typical root locus plot corresponding to OIFF is given in Figure 5(b). Note that the zero location changes with respect to $\beta$. The equivalent controller $\widehat{C}_{d}(s)$ can be written as

$$
\widehat{C}_{d}(s)=\frac{C_{d 2}(s)}{1+C_{d 2}(s) \beta},
$$

as shown in Figure 3(c). The modification amounts to replacing the integral controller with a first-order low-pass filter. Although the additional complexity is negligible, the damping performance is significantly improved. This result allows integral force feedback control to be applied to systems that were not previously suited.

\section{Tracking Control}

\subsection{Integral Control with Displacement Feedback}

The most straightforward technique for achieving displacement tracking is to simply enclose the system in an integral

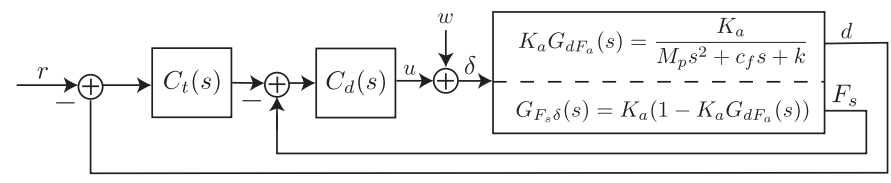

Figure 6: Tracking Loop.

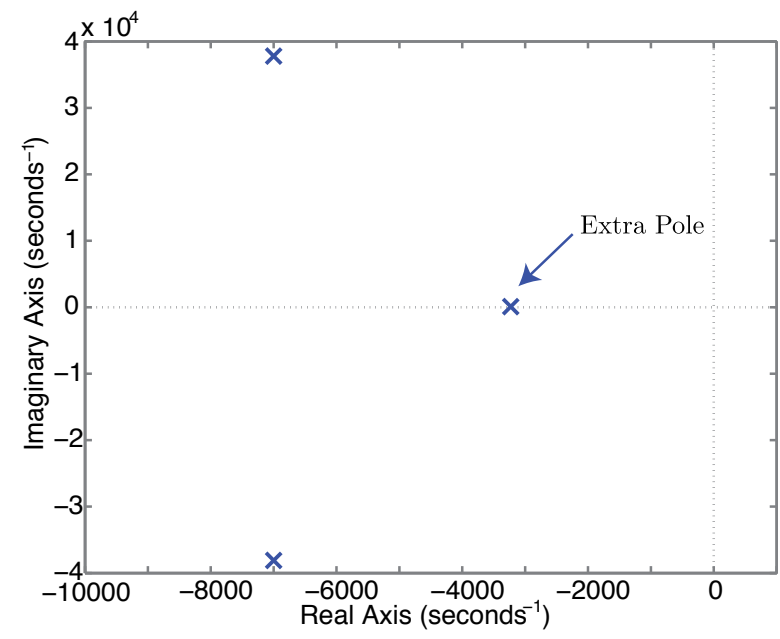

Figure 7: Pole-zero map of the Damping Loop.

feedback loop, as depicted in Figure 6. The tracking controller $C_{t 1}(s)$ is simply

$$
C_{t 1}(s)=\frac{K_{t 1}}{s}
$$

In this strategy, the displacement, $d$, must be obtained with a physical displacement sensor such as a capacitive, inductive, or optical sensor [26]. However, from the pole-zero map shown in Figure 7, the damped system contains a pair of resonance poles, plus an additional real axis pole due to OIFF. The additional pole unnecessarily increases the system order and reduces the achievable tracking bandwidth due to low-phase margin.

The characteristic equation of the closed-loop transfer function is given by the numerator of

$$
1+\frac{K_{t 1} K_{d 1}}{\gamma_{4} s^{4}+\gamma_{3} s^{3}+\gamma_{2} s^{2}+\gamma_{1} s^{1}}
$$

where

$$
\begin{aligned}
& \gamma_{4}=K_{a} M_{p}+M_{p} \beta, \\
& \gamma_{3}=c_{f} K_{a}+K_{a} K_{d 1} M_{p}+c_{f} \beta+K_{d 1} M_{p} \beta, \\
& \gamma_{2}=k K_{a}+c_{f} K_{a} K_{d 1}+k \beta+c_{f} K_{d 1} \beta, \\
& \gamma_{1}=k K_{a} K_{d 1}-K_{a}^{2} K_{d 1}+k K_{d 1} \beta .
\end{aligned}
$$

Proposition 1. Let $K_{d 1}$ and $K_{t 1}$ be the OIFF damping and integral tracking gain respectively. For a closed-loop system as implemented in Figure 6 to be stable, the gains must obey the following inequality: 


$$
K_{d 1} K_{t 1}<\frac{\left(K_{a}^{2}\left(-K_{a}^{2}+k\left(K_{a}+\beta\right)\right)\right)}{\left(M_{p}\left(K_{a}+\beta\right)\right)}
$$

Proof. In order to check the stability of the closed-loop transfer function, the zeros/numerator of (20) should be evaluated. The system is stable if all the zeros have negative real parts. Assuming that damping in the system is negligible, all necessary and sufficient conditions for stability are met if 1) all the coefficients of (20) are positive and 2) all the elements of the first column of the Routh-Hurwitz table are positive. The condition of stability is given as

$$
\begin{array}{r}
K_{d 1}^{3} K_{t 1} M_{p}\left(K_{a}+\beta\right)\left(K_{a} M_{p}+M_{p} \beta\right)+ \\
K_{d 1}^{2} M_{p}\left(K_{a}+\beta\right)\left(K_{a}^{4}-k K_{a}^{2}\left(K_{a}+\beta\right)\right)<0
\end{array}
$$

Rearranging (26) for $K_{t 1} K_{d 1}$ results in the expression (25).

\subsection{Structured PI Control with Displacement Feedback}

The location of the additional pole can be found by examining the characteristic equation (20) of the damped system. For the system under consideration, the roots of the characteristic equation contain a complex pair and a pole on the real axis as shown before.

To eliminate the additional pole from the tracking loop, the controller can be parameterised so that it contains a zero at the same frequency. A controller that achieves this is

$$
C_{t 2}(s)=\frac{K_{t 2}(s+p)}{s p}
$$

where $p$ is the location of the additional pole identified by finding the roots of (20) using Cardano's method [27],

$$
\begin{aligned}
p & =-(A+B-a / 3), \\
a & =K_{d 2}+\frac{c_{f}}{M_{p}}, \\
b & =\frac{k+c_{f} K_{d 2}}{M_{p}}, \\
c & =\frac{K_{d 2}\left(-K_{a}^{2}+k\left(K_{a}+\beta\right)\right)}{M_{p}\left(K_{a}+\beta\right)}, \\
Q & =\frac{a^{2}-3 b}{9}, R=\frac{2 a^{3}-9 a b+27 c}{54}, \\
A & =-\sqrt[3]{R+\sqrt[2]{R^{2}-Q^{3}}}, B=Q / A .
\end{aligned}
$$

The integral gain is chosen in the normal way to provide the desired stability margins. The form of this controller is identical to a PI controller except that the zero location is fixed. This is advantageous since the controller has only one free tuning parameter.

\section{Example System}

Here, we examine a single degree of freedom positioner based on the mechanical diagram shown in Figure 2 with mass $M_{p}=250 \mathrm{~g}$, flexure stiffness $k_{f}=300 \mathrm{~N} / \mu \mathrm{m}$, actuator stiffness

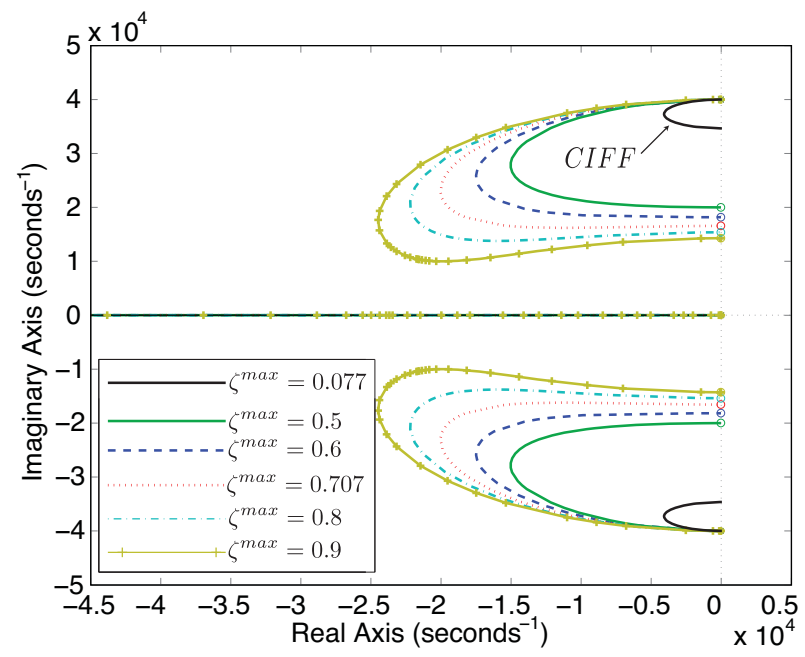

Figure 8: Example System: Root locus comparison between CIFF and OIFF.

Table 1: Comparison between analytic and numerically obtained damping ratio $\zeta^{\text {max }}$ and feedback gain $K_{d 2}$ for the example system.

\begin{tabular}{|c|c|c|c|c|}
\cline { 2 - 5 } \multicolumn{1}{c|}{} & \multicolumn{2}{c|}{ Analytic } & \multicolumn{2}{c|}{ Numerical } \\
\hline$\beta$ & $\zeta^{\max }$ & $K_{d 2}$ & $\zeta^{\max }$ & $K_{d 2}$ \\
\hline$-6.67 \times 10^{7}$ & 0.500 & $5.65 \times 10^{4}$ & 0.501 & $5.57 \times 10^{4}$ \\
$-6.98 \times 10^{7}$ & 0.707 & $6.21 \times 10^{4}$ & 0.708 & $6.23 \times 10^{4}$ \\
$-7.13 \times 10^{7}$ & 0.900 & $6.69 \times 10^{4}$ & 0.902 & $6.70 \times 10^{4}$ \\
\hline
\end{tabular}

$K_{a}=100 \mathrm{~N} / \mu \mathrm{m}$ and flexure damping $c_{f}=10 \mathrm{~N} / \mathrm{ms}^{-1}$. The frequency of the open-loop poles and zeros of the system are

$$
\omega_{1}=6.37 \mathrm{kHz} \quad z_{1}=5.5 \mathrm{kHz}
$$

The optimal gain and maximum damping ratio for the example system using CIFF is $K_{d 1}=4.3 \times 10^{4}$ and $\zeta_{1}^{\max }=0.077$. The numerically obtained optimal gain is $4.57 \times 10^{4}$ and the damping ratio is 0.077 . These values are obtained from the root-locus plot shown in Figure 8 and correlate closely with the predicted values which supports the accuracy of the assumptions made in deriving the optimal gain. With OIFF, the relationship between $\beta$ and $\zeta$ is described in (17) and plotted in Figure 9. The maximum modal damping with CIFF is 0.077; however, with OIFF, the maximum modal damping can be varied from 0.077 to 1 at different values of $\beta$.

The root locus of the system is shown in Figure 8. The optimal feedback gain, maximum damping ratio and corresponding value of $\beta$ is given in Table 1 . These values can be validated by the numerical root-locus plot in Figure 8 and are summarized in Table 1.

If a disturbance, $w$, is added into the system. The transfer function from the disturbance, $w$, to the sensor force, $F_{s}$, is

$$
G_{F_{s} w}(s)=\frac{F_{s}}{w}=\frac{G_{F_{s}} \delta}{1+C_{d} G_{F_{s}} \delta} .
$$

where $C_{d}=C_{d 1}$ for CIFF and $C_{d}=\widehat{C}_{d}$ for OIFF. The simulated open-loop and closed-loop frequency responses of (29) are plotted in Figure 10 for both CIFF and OIFF. The transfer function 


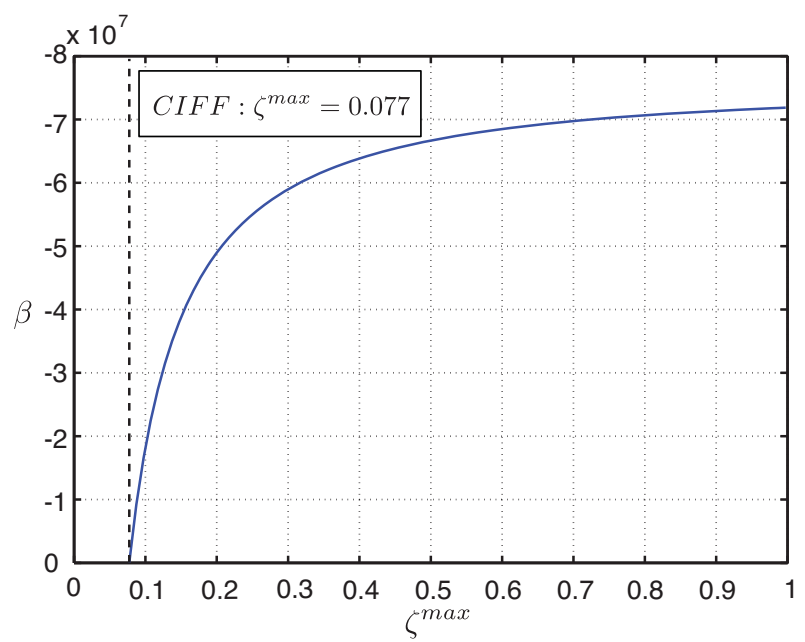

Figure 9: Example System: The relationship between $\beta$ and $\zeta$ for OIFF

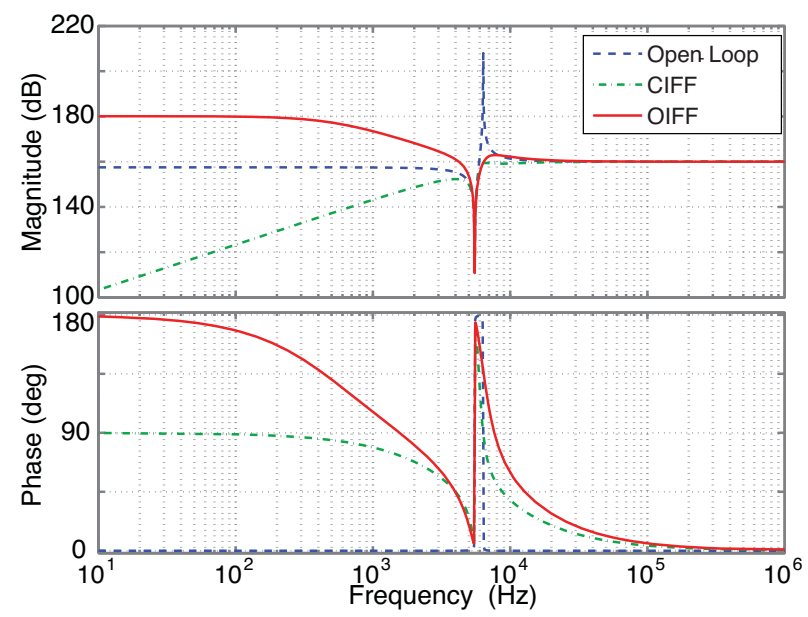

Figure 10: Example System: Frequency response from the input disturbance $w$ to the sensor force $F_{S}$

from the disturbance $w$ to the displacement of the platform $d$ are given as

$$
G_{d w}(s)=\frac{d}{w}=\frac{K_{a} G_{d F_{a}}}{1+C_{d} G_{F_{s}} \delta} .
$$

The simulated open-loop and closed-loop frequency responses of (30) for both cases are shown in Figure 11. For the OIFF case, the closed-loop transfer function measured from the reference, $r$, to the sensor force, $F_{s}$, is

$$
G_{F_{s}}(s)=\frac{F_{s}}{r}=\frac{C_{d} G_{F_{s} \delta}}{1+C_{d} G_{F_{s}} \delta} .
$$

when $s=0$

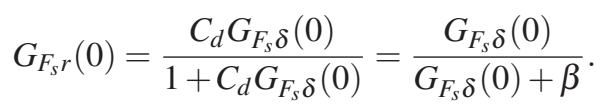

This shows that the DC gain of the closed-loop increases as $\beta$ is decreased. Recall that the maximum damping ratio of the closed-loop system increases as $\beta$ is decreased.

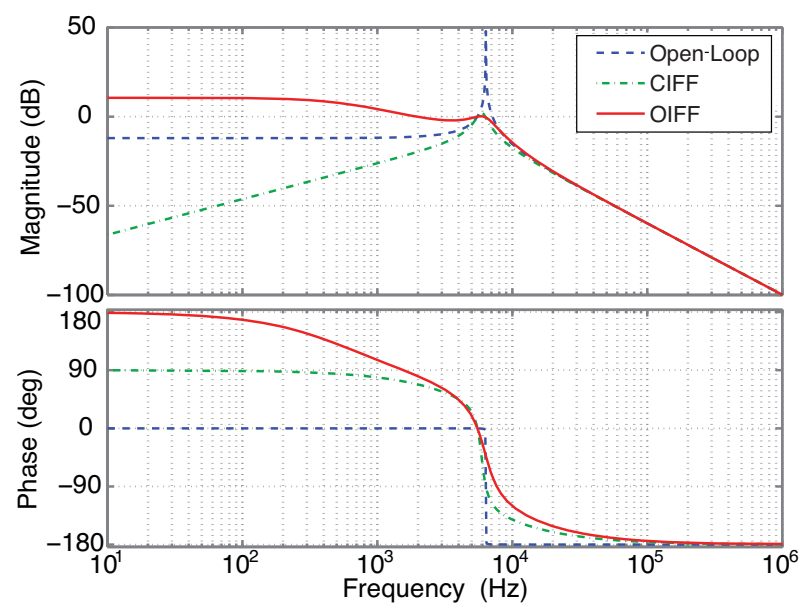

Figure 11: Example System: Frequency response from the input disturbance $w$ to the displacement of the platform $d$.

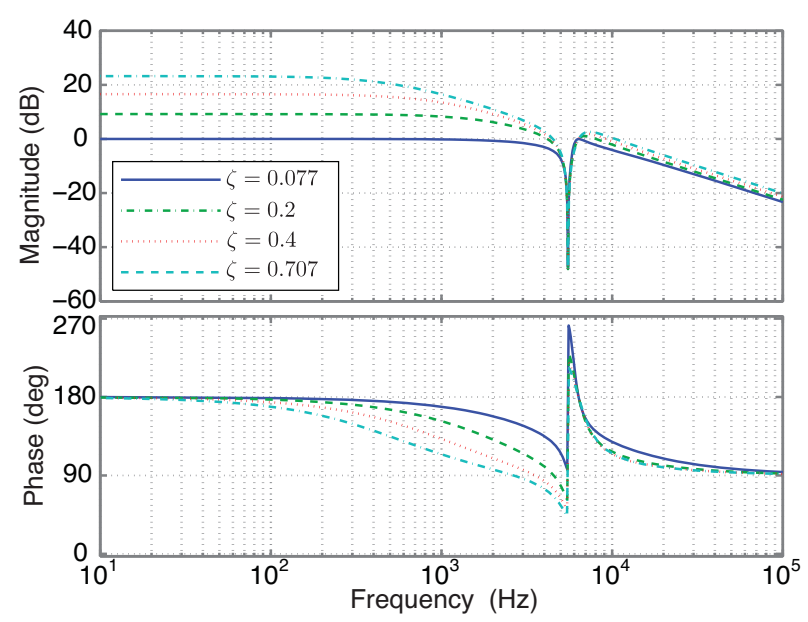

Figure 12: Example System: Frequency response from the input disturbance $w$ to the control action $u$ as $\beta$ is increased.

In addition, the effect of control action with respect to input disturbance is examined. The transfer function between the input disturbance $w$ to the control action $u$ is

$$
G_{u w}(s)=\frac{u}{w}=\frac{C_{d} G_{F_{s}} \delta}{-1-C_{d} G_{F_{s}} \delta} .
$$

The frequency response of this transfer function is shown in Figure 12. The sensitivity of the control action toward input disturbance increases as the desired damping ratio is increased.

We now examine the performance of the tracking controllers. The example system is damped using OIFF with a desired damping ratio of 0.707 . With the basic integral tracking control, the closed-loop bandwidth of the system is $400 \mathrm{~Hz}$ with a gain margin of $20 \mathrm{~dB}$. As expected, the closed-loop bandwidth is limited by low-phase margin. The corresponding damping and tracking gain are $6.21 \times 10^{4}$ and $4.0 \times 10^{10}$. The product of the two gains satisfies the stability condition given in (25). However, with the structured PI tracking controller, the closed-loop band- 


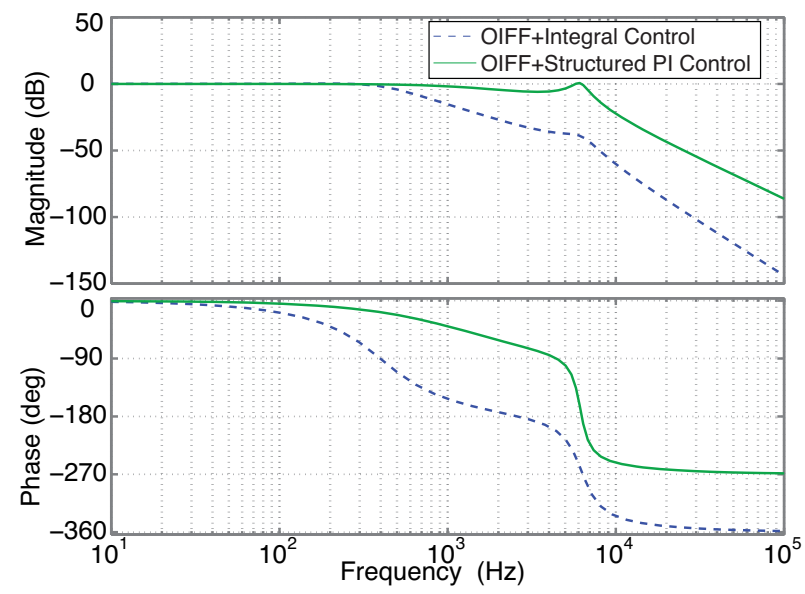

Figure 13: Example System: Closed-loop frequency responses from the reference $r$ to the displacement of the platform $d$ of the system.

width of the system is increased to $1200 \mathrm{~Hz}$ with a phase-margin of 60 degrees. This shows an improvement of threefold just by cancelling an additional real pole induced by the inner damping loop. Figure 13 shows the closed-loop frequency response of the system using the two tracking control architecture discussed previously.

To examine the constant velocity tracking performance, a triangular waveform was applied as a reference with a frequency of $200 \mathrm{~Hz}$. Figure 14(a) shows the displacement of the system. The tracking error is plotted in Figure 14(b), The optimal force feedback controller can be observed to heavily reduce the tracking error.

\section{Effect of Higher Order Modes}

So far, only a single degree of freedom system has been considered. Although this is appropriate for modelling the first resonance mode, it does not capture the higher order modes that occur in distributed mechanical systems. However, the higher order modes do not disturb the zero-pole ordering of the transfer function from the applied actuator voltage to the measured force. To illustrate this concept, we augment the open-loop system with an additional second-order system at five times the resonance frequency of the first mode. Figure 15 shows the closed-loop frequency responses of the system. Note that the second mode does not affect the performance of the system.

The transfer function of a generalized mechanical system with a discrete piezoelectric transducer and collocated force sensor is guaranteed to exhibit zero-pole ordering as shown in [22]. That is, the transfer function $G_{F_{S} F_{a}}$ will always exhibit zero-pole ordering. As the zero-pole ordering of the system is guaranteed, it follows that the controller discussed in the previous section will also guarantee the stability of systems with multiple modes.

\section{Application to Objective Lens Positioner}

The experiment was conducted on a Queensgate OSM-Z100B objective lens positioner with 3 different objective loads

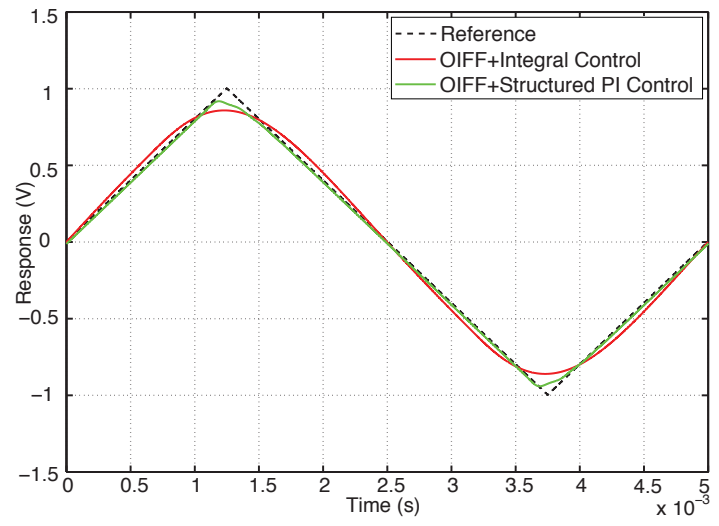

(a) Position

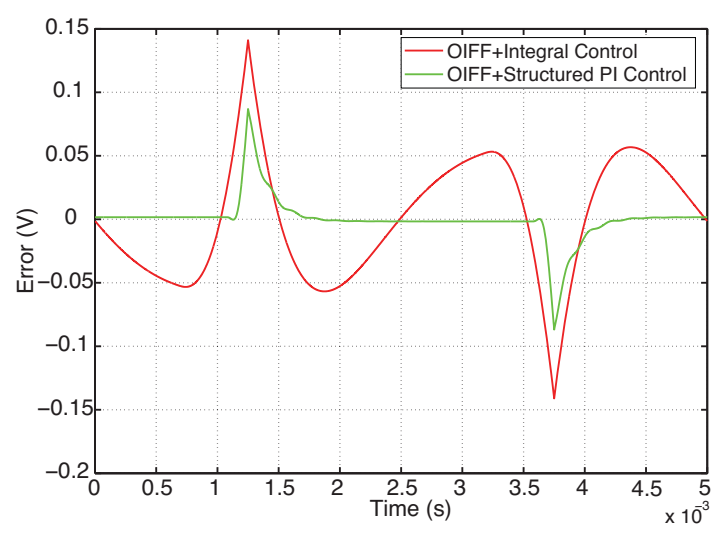

(b) Error

Figure 14: Example System: Constant velocity tracking with triangular wave.

as shown in Figure 16. This single-axis lens positioner has a range of $100 \mu \mathrm{m}$ and a static stiffness of $1.5 \mathrm{~N} / \mathrm{m}$. The inner loop damping controller is implemented using analog electronics. The outer tracking loop is implemented using a Queensgate NPS4110 controller. The block diagram of the experimental setup is shown in Figure 17.

By referring to set-up in Figure 17, the open-loop frequency response of the positioner with $100 \times$ objective was measured from the voltage amplifier input $u_{2}$ which is proportional to the internal actuator force, $F_{a}$, to the force sensor, $F_{s}$, and position sensor output, $d$, with an excitation of $100 \mathrm{mV}_{\mathrm{pp}}$ random noise signal. The open-loop frequency responses are shown in Figure 18 which exhibits a resonance frequency at around $383 \mathrm{~Hz}$. The first two modes are relatively close in frequency. The system can be approximated by a second-order transfer function given as

$$
G_{F_{s} F_{a}}(s)=\frac{2.141 s^{2}+736.4 s+6.072 \times 10^{6}}{s^{2}+214 s+5.606 \times 10^{6}}
$$

\subsection{Damping Controller Design}

The optimal gain and maximum damping ratio of system (34) using CIFF are $K_{d 1}=1500$ and $\zeta_{1}^{\max }=0.3$. These values can also be obtained numerically from the root locus plot in Figure 19. The numerically found optimal gain is 1700 and the corresponding damping ratio is 0.33 . Figure 19 also includes the 


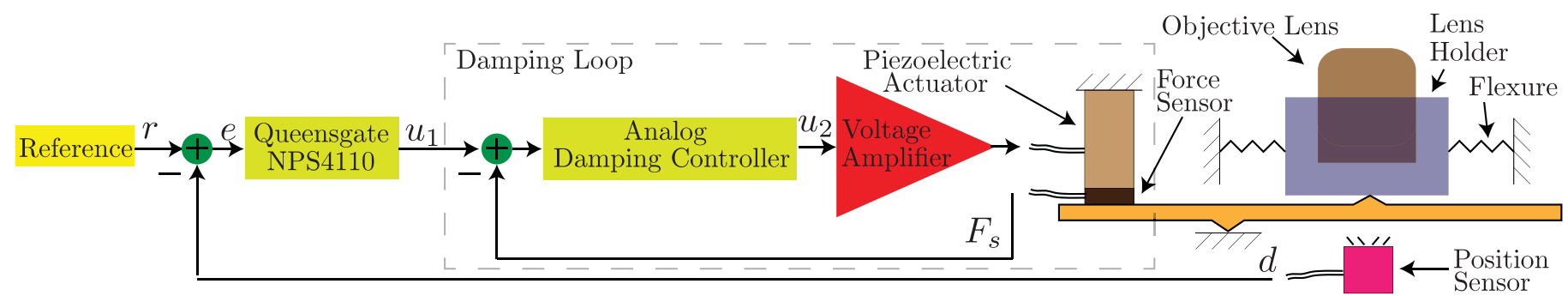

Figure 17: Block diagram of the experimental setup.

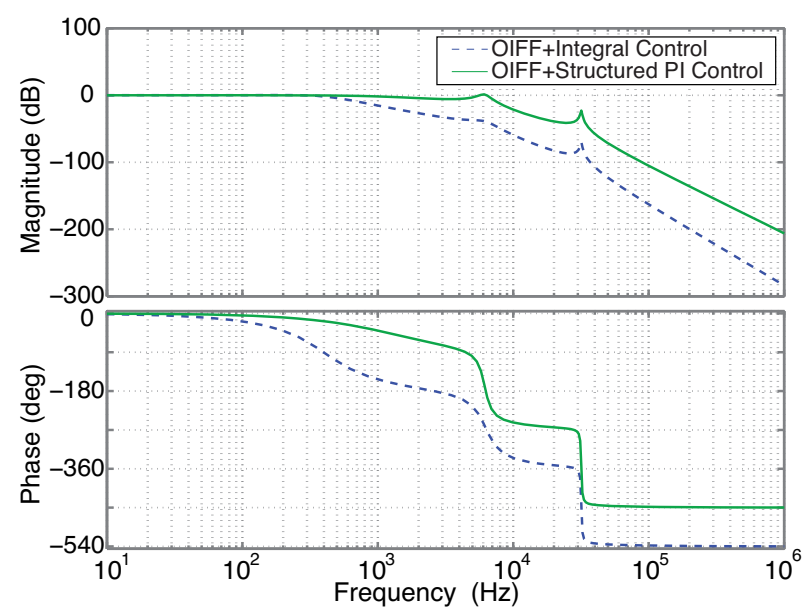

Figure 15: Example System: Closed-loop frequency responses from the reference $r$ to the displacement of the platform $d$ of the system with higher order model.

Table 2: Experimental results: Numerically obtained data for OIFF.

\begin{tabular}{|c|c|c|c|c|c|}
\hline$\beta$ & $\zeta_{i}^{\text {max }}$ & $K_{d 2}$ & $\beta$ & $\zeta_{i}^{\text {max }}$ & $K_{d 2}$ \\
\hline 0.0 & 0.33 & 1700 & -0.4 & 0.49 & 2350 \\
-0.1 & 0.36 & 1840 & -0.5 & 0.57 & 2500 \\
-0.2 & 0.39 & 2060 & -0.6 & 0.68 & 2840 \\
-0.3 & 0.44 & 2160 & -0.7 & 0.85 & 3300 \\
\hline
\end{tabular}

root locus plots of the system using OIFF with different feedthrough terms $\beta$. The relationship between $\beta$ and $\zeta^{\max }$ (shown in Figure 20) was numerically obtained from the root locus plot and summarised in Table 2. The maximum damping ratio is increased from 0.33 (CIFF) to 0.85 by just adjusting the value of $\beta$. Figure 18 includes the closed-loop frequency responses of the system using OIFF with $\beta=-0.6$. The closed-loop frequency responses are measured using the same procedure as the open-loop responses. The closed-loop response shows that the first and second resonance modes have been effectively eliminated. The higher frequency modes have also been damped by up to $5 \mathrm{~dB}$.

\subsection{Tracking Control Design}

The performance of the commercial PID controller was tuned experimentally as there was no direct access to the PID parameters on the commercial controller. The tuning minimized the settling time due to a step input reference. The implemented

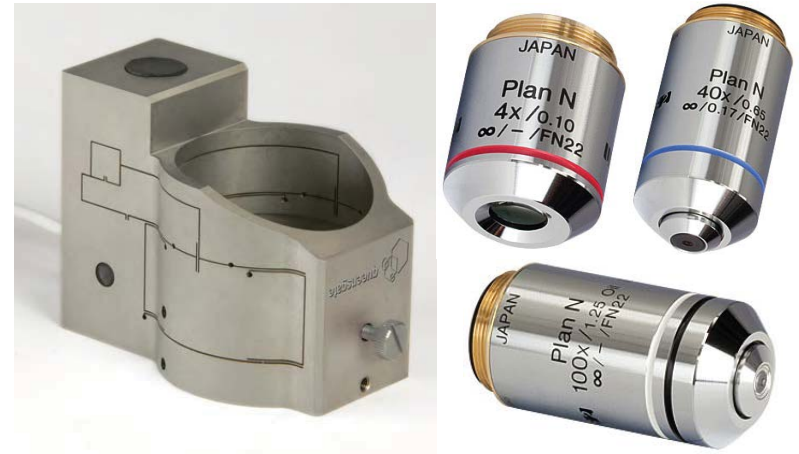

Figure 16: Queensgate OSM-Z-100B objective lens positioner (Left) and Olympus $4 \times, 40 \times$ and $100 \times$ objective lens (Right).

PID controller has the following structure

$$
C_{t}(s)=k_{p}+\frac{k_{i}}{s}+\frac{k_{d} s}{\tau_{d} s+1}
$$

where $k_{p}=0.01, k_{i}=2000, k_{d}=1 \times 10^{-6}$ and $\tau_{d}=1.25 \times 10^{-7}$. The derivative component is an approximation that facilitates practical implementation. The approximation acts as a derivative at low frequency, while reducing the gain at high frequency with an additional pole [28]. The term $\tau_{d}$ limits the gain,hence, the high-frequency signal is amplified at most by a factor of $k_{d} / \tau_{d}=8$.

The structured PI controller is

$$
C_{t}(s)=\frac{700(s+2914)}{2914 s}
$$

where $s=-2914$ is the location of the additional pole. The only tuning parameter here is $K_{t}$ which was tuned to provide acceptable stability margins. The loop return ratio plot of the controllers are shown in Figure 21. The gain and phase margin of the system with commercial PID control is $3.2 \mathrm{~dB}$ and 86 degrees respectively. The gain and phase margin of the system with Optimal Integral Force Feedback and Structured PI control is $17.3 \mathrm{~dB}$ and 75 degrees respectively.

The closed-loop frequency responses of the standard commercial controller and the proposed controller are plotted in Figure 22. The achievable tracking bandwidth of the commercial PID controller is $26.1 \mathrm{~Hz}$ compared to $255 \mathrm{~Hz}$ with the proposed controller. 

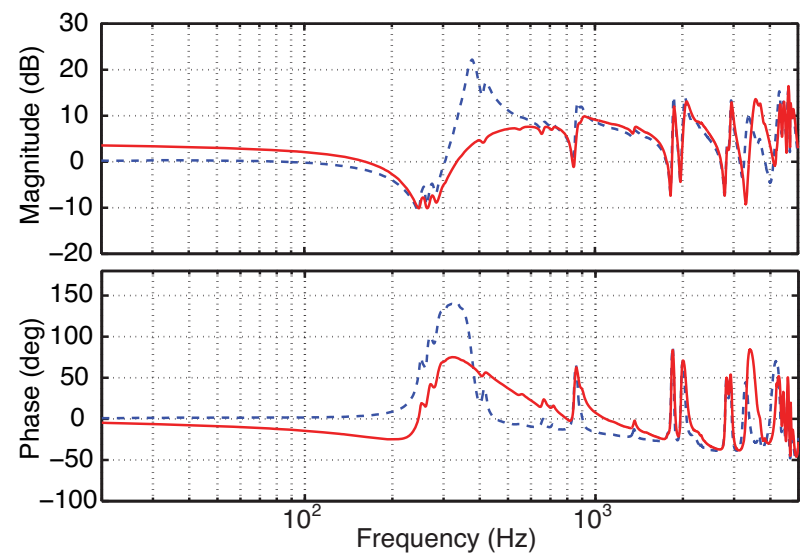

(a) Open-loop (dashed line) and closed-loop (solid line) frequency response measured from $u_{2}$ to the output of the sensor force.
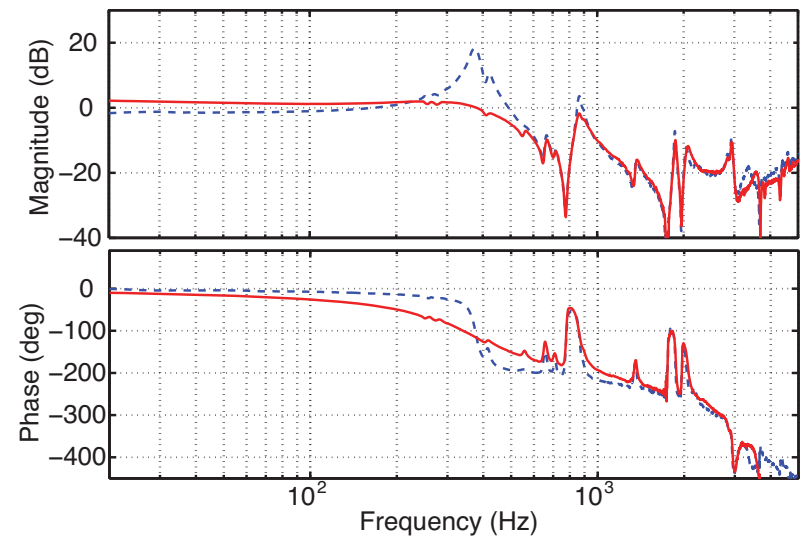

(b) Open-loop (dashed line) and closed-loop (solid line) frequency response measured from $u_{2}$ to the output of the position sensor.

Figure 18: Experimental Results: Open-loop and closed-loop frequency responses using CIFF and OIFF.

\subsection{Sensitivity to Variations in Resonance Frequency}

The force feedback loop was initially tuned for the $100 \times$ objective which has a nominal mass of $88.8 \mathrm{~g}$. In order to show the insensitivity to variation in resonance frequency, other objectives were considered without retuning the tracking loop. The objectives are $4 \mathrm{x}$ objective $(47.8 \mathrm{~g}$ ) and a modified $40 \mathrm{x}$ objective $(163.3 \mathrm{~g})$. The variations in resonance frequency and performance are summarized in Table 3. The open-loop and force feedback response of the stage with different loads are plotted in Figure 23 and Figure 24. It can be observed that the tuning of the force feedback loop is not sensitive to changes in resonance frequency. The tracking controller frequency responses with, and without force feedback are plotted in Figure 25 and Figure 26. As expected from the results in Figure 24, the only significant change in the force feedback response is the bandwidth, which is proportional to the resonance frequency.

\subsection{Constant Velocity Tracking and Step Response}

To examine the constant velocity tracking performance, a $80 \mu \mathrm{m}$ sawtooth waveform was applied as a reference with a frequency of $3 \mathrm{~Hz}$ and at $95 \%$ duty cycle. Figure 27(a) shows

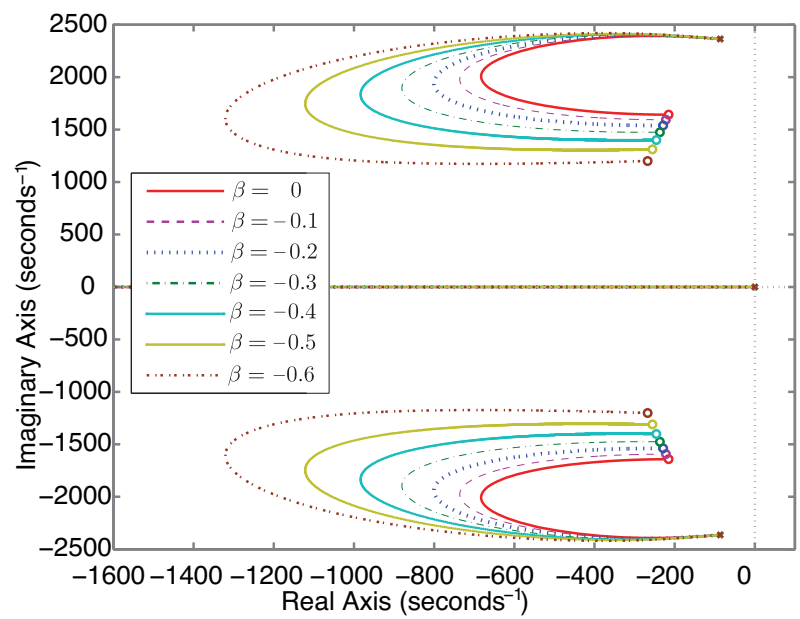

Figure 19: Experimental results: Root locus using OIFF.

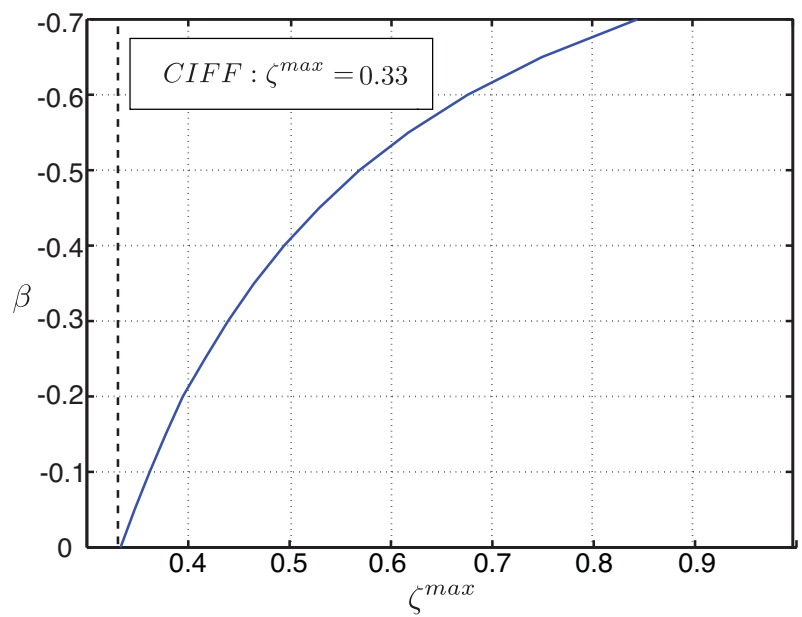

Figure 20: Experimental results: Relationship between $\zeta^{\max }$ and $\beta$ using OIFF.

the displacement of the system. The tracking error is plotted in Figure 27(b), The optimal force feedback controller can be observed to reduce the tracking error by upto $65 \%$.

The step responses for different objective masses are also plotted in Figure 28. The heavier objective requires a proportionally longer settling time. The settling time of the system with the proposed damping and tracking controller is greatly improved compared to the standard PID controller.

Table 3: Experimental results: Influence of objective mass on performance

\begin{tabular}{|l|r|r|r|}
\hline & $4 \times$ & $100 \times$ & $40 \times+$ mass \\
\hline Mass & $47.8 \mathrm{~g}$ & $88.8 \mathrm{~g}$ & $163.3 \mathrm{~g}$ \\
Resonance Freq. & $412 \mathrm{~Hz}$ & $378 \mathrm{~Hz}$ & $264 \mathrm{~Hz}$ \\
Force Feedback BW & $500 \mathrm{~Hz}$ & $398 \mathrm{~Hz}$ & $326 \mathrm{~Hz}$ \\
Tracking BW (PID) & $31.6 \mathrm{~Hz}$ & $26.1 \mathrm{~Hz}$ & $21.5 \mathrm{~Hz}$ \\
Tracking BW (OIFF) & $167 \mathrm{~Hz}$ & $255 \mathrm{~Hz}$ & $212 \mathrm{~Hz}$ \\
\hline
\end{tabular}




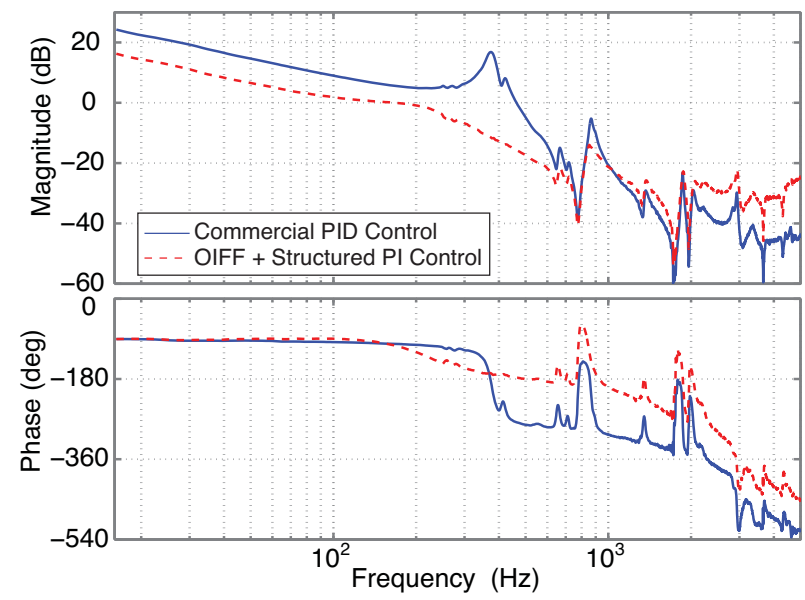

Figure 21: Experimental Result: Comparison between the loop return ratio plots of the system with a commercial PID controller (solid line) and Optimal Integral Force Feedback and Structured PI Control (dashed line).

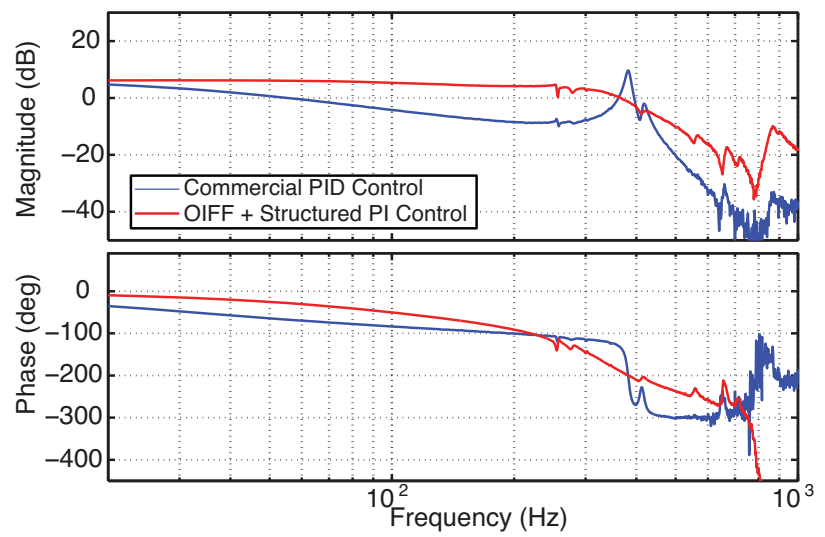

Figure 22: Experimental Result: Closed-loop frequency response of the tracking loop measured from $r$ to the position sensor output $d$, scaled to um/V.

\section{Conclusions}

The maximum damping achievable with classical Integral Force Feedback control is limited by the frequency difference between the systems poles and zeros. This paper describes a novel improvement that allows an arbitrary damping ratio to be achieved for any system by introducing an additional feedthrough term. For systems with closely spaced poles and zeros, the damping performance may be significantly improved.

The second contribution is a structured PI controller for tracking loop of systems with force feedback. The proposed tracking controller is parameterized so that it contains a zero that cancel the additional pole due introduced by the damping controller. This approach improves the system phase margin and closed-loop servo bandwidth.

The proposed techniques are demonstrated on an objective lens positioning system. With a commercial PID controller, the maximum tracking bandwidth is $26.1 \mathrm{~Hz}$. However, with the proposed tracking and damping controller, the tracking bandwidth is increased to $255 \mathrm{~Hz}$.

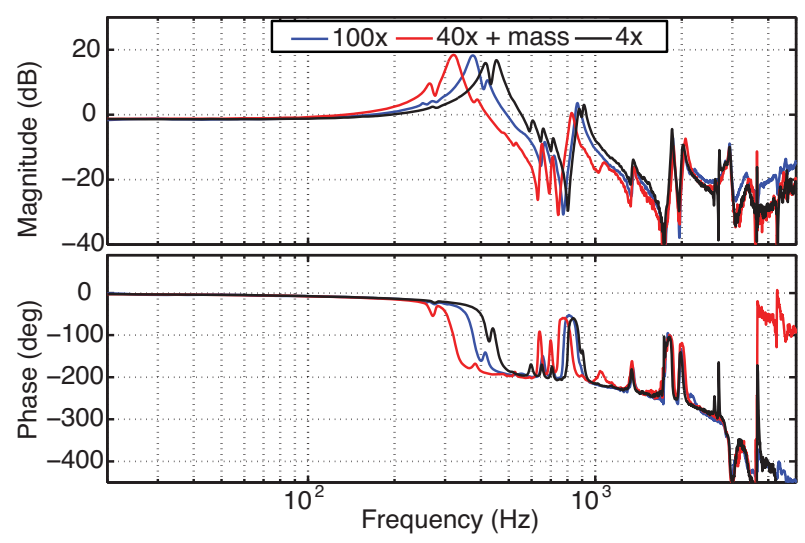

Figure 23: Experimental Results: Open-loop frequency response measured from $u_{2}$ to the output of the position sensor output $d$, scaled to um/V with different objectives.

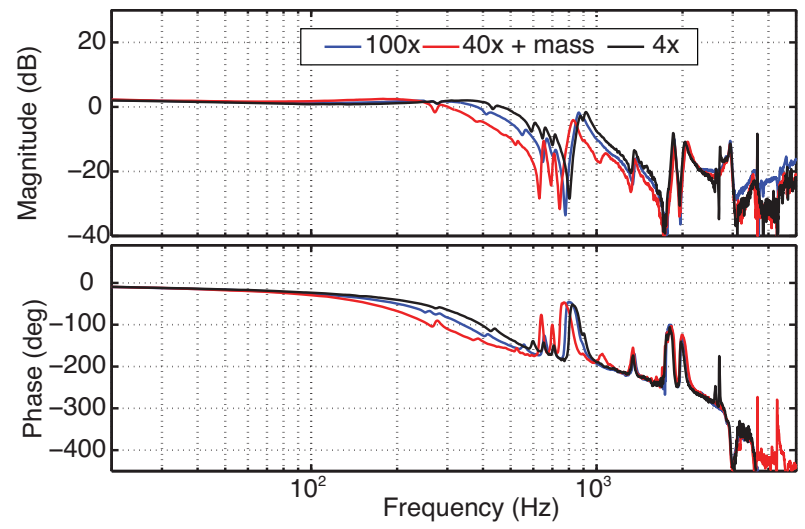

Figure 24: Experimental Results: Closed-loop frequency response of the damping loop measure from $u_{2}$ to the output of the position sensor output $d$, scaled to $\mathrm{um} / \mathrm{V}$ with different objectives.

\section{References}

[1] D. Semwogerere, E. R. Weeks, Confocal microscopy, Encyclopedia of Biomaterials and Biomedical Engineering. G. Wnek, and G. Bowlin, editors. Taylor and Francis, New York.

[2] S. M. Salapaka, M. V. Salapaka, Scanning probe microscopy, IEEE Control Systems 28 (2) (2008) 65-83.

[3] K. K. Leang, A. J. Fleming, High-speed serial-kinematic AFM scanner: design and drive considerations, Asian Journal of Control 11 (2) (2009) 144-153. doi:10.1002/asjc.090.

[4] A. A. Tseng, A. Notargiacomob, T. P. Chen, Nanofabrication by scanning probe microscope lithography: A review, Journal of Vacuum Science and Technology 23 (3) (2005) 877-894.

[5] R. A. Oliver, Advances in afm for the electrical characterization of semiconductors, Reports on Progress in Physics 71 (7) (2008) 076501

[6] A. J. Fleming, Nanopositioning system with force feedback for highperformance tracking and vibration control, IEEE Transactions on Mechatronics 15 (3) (2010) 433-447.

[7] A. A. Eielsen, M. Burger, J. T. Gravdahl, K. Y. Pettersen, $\mathrm{PI}^{2}$-controller applied to a piezoelectric nanopositioner using conditional integrators and optimal tuning, in: Proc. IFAC World Congress, Vol. 18, Milano, 2011. doi:10.3182/20110828-6-IT-1002.01401.

[8] D. Y. Abramovitch, S. Hoen, R. Workman, Semi-automatic tuning of PID gains for atomic force microscopes, in: Proc. American Control Conference, Seattle, WA, 2008, pp. 2684-2689.

[9] A. Sebastian, S. M. Salapaka, Design methodologies for robust nanopositioning, IEEE Transactions on Control Systems Technology 13 (6) (2005) 868-876. doi:10.1109/TCST.2005.854336. 


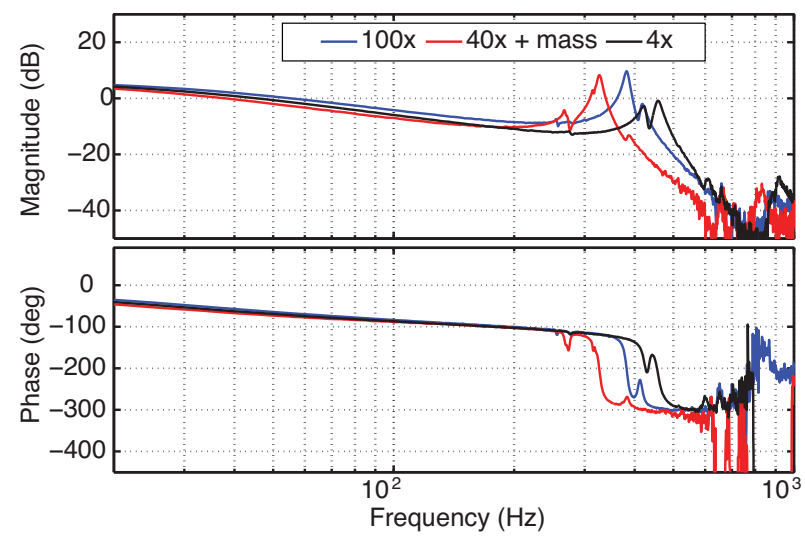

Figure 25: Experimental Results: Closed-loop frequency response of the tracking loop measured from $r$ to the output of the position sensor output $d$, scaled to um/V, with a commercial PID controller.

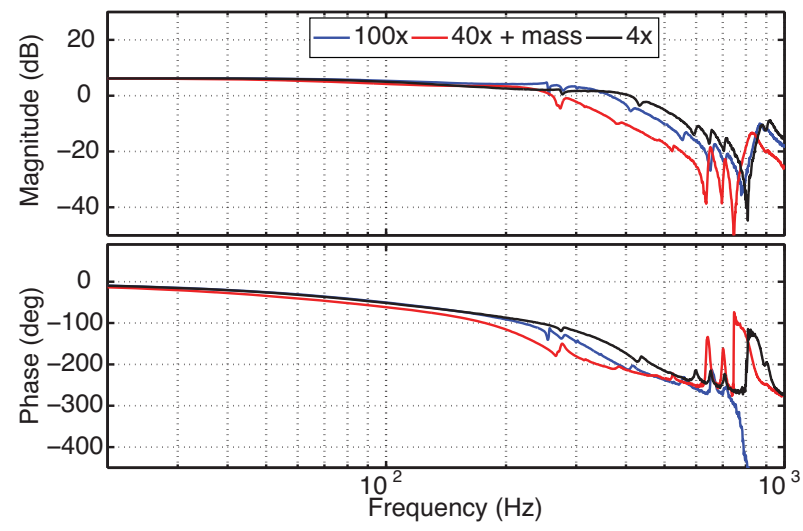

Figure 26: Experimental Results: Closed-loop frequency response of the tracking loop measured from $r$ to the position sensor $d$, scaled to um/V, with Optimal Integral Force Feedback and Structured PI control.

[10] S. Kuiper, G. Schitter, Active damping of a piezoelectric tube scanner using self-sensing piezo actuation, Mechatronics 20 (6) (2010) 656 - 665 .

[11] S. Devasia, E. Eleftheriou, S. O. R. Moheimani, A survey of control issues in nanopositioning, IEEE Transactions on Control Systems Technology 15 (5) (2007) 802-823.

[12] J. L. Fanson, T. K. Caughey, Positive position feedback control for large space structures, AIAA Journal 28 (4) (1990) 717-724.

[13] S. S. Aphale, B. Bhikkaji, S. O. R. Moheimani, Minimizing scanning errors in piezoelectric stack-actuated nanopositioning platforms, IEEE Transactions on Nanotechnology 7 (1) (2008) 79-90.

[14] S. N. Mahmoodi, M. Ahmadian, Modified acceleration feedback for active vibration control of aerospace structures, Smart Materials and Structures 19 (6) (2010) 065015.

[15] A. J. Fleming, S. O. R. Moheimani, Sensorless vibration suppression and scan compensation for piezoelectric tube nanopositioners, IEEE Transactions on Control Systems Technology 14 (1) (2006) 33-44.

[16] A. J. Fleming, S. Behrens, S. O. R. Moheimani, Optimization and implementation of multi-mode piezoelectric shunt damping systems, IEEE/ASME Transactions on Mechatronics 7 (1) (2002) 87-94.

[17] H. R. Pota, S. O. R. Moheimani, M. Smith, Resonant controllers for smart structures, in: Proc. IEEE Conference on Decision and Control, Phoenix, Arizona, 1999, pp. 631-636.

[18] S. S. Aphale, A. J. Fleming, S. O. R. Moheimani, Integral resonant control of collocated smart structures, IOP Smart materials and Structures 16 (2007) 439-446. doi:10.1088/0964-1726/16/2/023.

[19] A. J. Fleming, S. S. Aphale, S. O. R. Moheimani, A new method for robust damping and tracking control of scanning probe microscope posi-

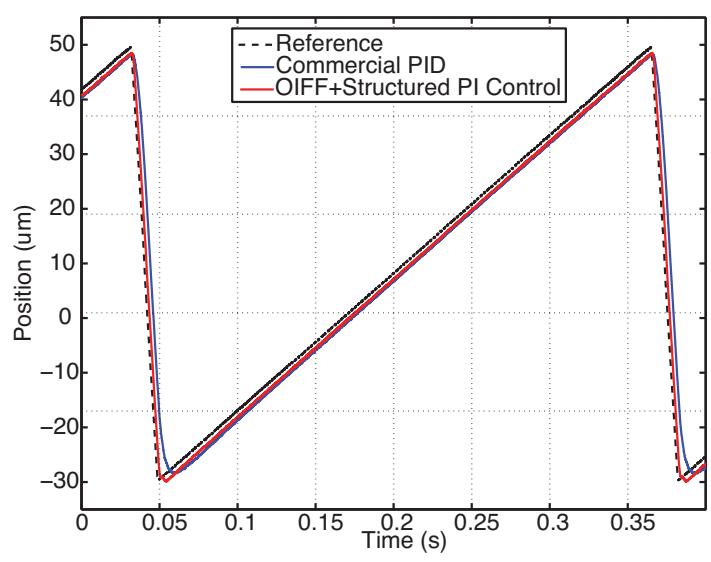

(a) Position

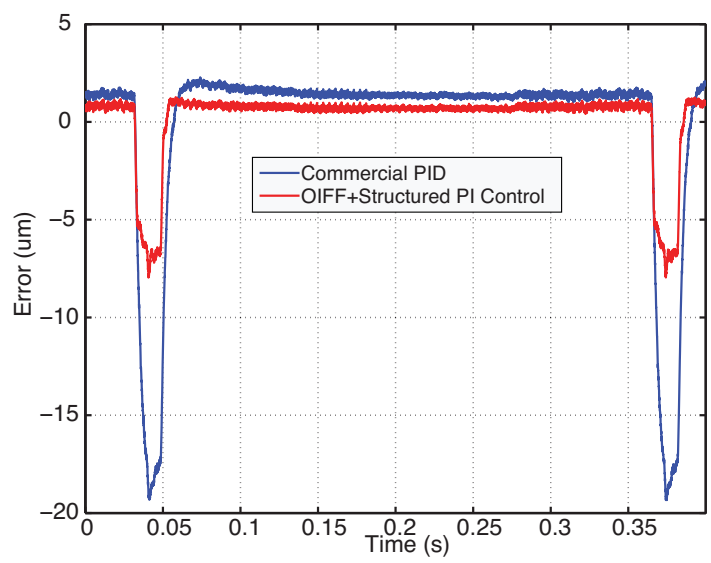

(b) Error

Figure 27: Experimental results: Constant velocity tracking of a $80 \mu \mathrm{m}$ sawtooth waveform with a frequency of $3 \mathrm{~Hz}$ and at $95 \%$ duty cycle.

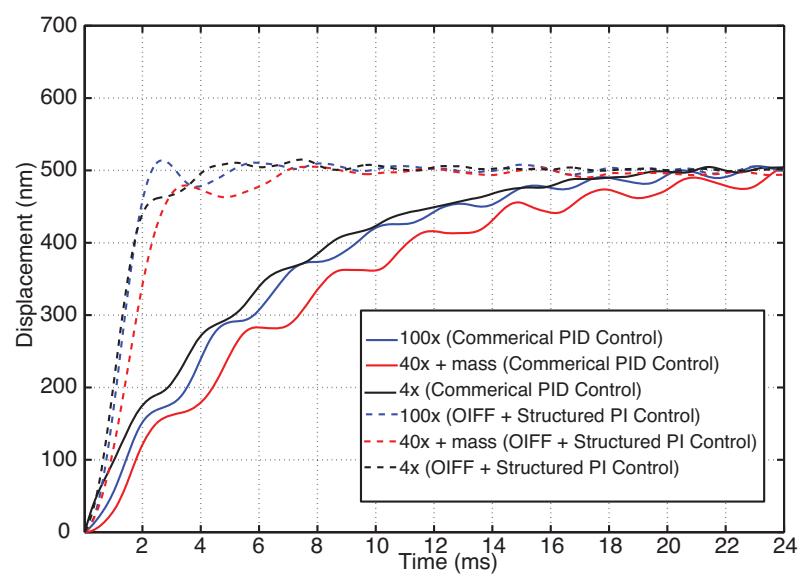

Figure 28: Experimental results: Closed-loop step response comparison between different objectives and controllers. The step reference is $500 \mathrm{~nm}$.

tioning stages, IEEE Transactions on Nanotechnology 9 (4) (2010) 438448. doi:10.1109/TNANO.2009.2032418.

[20] I. R. Petersen, A. Lanzon, Feedback control of negativeimaginary systems, Control Systems, IEEE 30 (5) (2010) $54-72$. doi:10.1109/MCS.2010.937676.

[21] A. Preumont, J. P. Dufour, C. Malekian, Active damping by a local force feedback with piezoelectric actuators., AIAA Journal of Guidance and 
Control 15 (2)

[22] A. Preumont, B. de Marneffe, A. Deraemaeker, F. Bossens, The damping of a truss structure with a piezoelectric transducer, Computers and Structures 86.

[23] A. Preumont, Vibration control of active structures, Kluwer Academic Publishers, 2002.

[24] A. Preumont, Mechatronics, Dynamics of electromechanical and piezoelectric systems, Springer, Dordrecht, The Netherlands, 2006.

[25] A. J. Fleming, K. K. Leang, Integrated strain and force feedback for high performance control of piezoelectric actuators, Sensors and Actuators A 161 (1-2) (2010) 256-265. doi:10.1016/j.sna.2010.04.008.

[26] A. J. Fleming, A review of nanometer resolution position sensors: Operation and performance, Sensors and Actuators A: Physical 190 (2013) 106-126.

[27] W. H. Press, S. A. Teukolsky, W. T. Vetterling, B. P. Flannery, Numerical recipes 3rd edition: The art of scientific computing, Cambridge university press, 2007.

[28] K. J. Aström, R. M. Murray, Feedback systems: an introduction for scientists and engineers, Princeton university press, 2010. 